\title{
Asymptotics and oscillation of higher-order functional dynamic equations with Laplacian and deviating arguments
}

\section{Taher S Hassan*}

\section{"Correspondence: \\ tshassan@mans.edu.eg \\ Department of Mathematics, \\ Faculty of Science, University of Hail, Hail, 2440, Saudi Arabia \\ Department of Mathematics, \\ Faculty of Science, Mansoura \\ University, Mansoura, 35516, Egypt}

$$
\begin{aligned}
& \text { Abstract } \\
& \text { In this paper, we deal with the asymptotics and oscillation of } \\
& \text { higher-order nonlinear dynamic equations with Laplacian and } \\
& \text { the form } \\
& \qquad\left\{r_{n-1}(t) \phi_{\alpha_{n-1}}\left[\left(r_{n-2}(t)\left(\cdots\left(r_{1}(t) \phi_{\alpha_{1}}\left[x^{\Delta}(t)\right]\right)^{\Delta} \cdots\right)^{\Delta}\right)^{\Delta}\right]\right\}^{\Delta} \\
& \qquad+\sum_{v=0}^{N} p_{v}(t) \phi_{\gamma_{v}}\left(x\left(g_{v}(t)\right)\right)=0
\end{aligned}
$$

In this paper, we deal with the asymptotics and oscillation of the solutions of higher-order nonlinear dynamic equations with Laplacian and mixed nonlinearities of

on an above-unbounded time scale. By using a generalized Riccati transformation and integral averaging technique we study asymptotic behavior and derive some new oscillation criteria for the cases without any restrictions on $g(t)$ and $\sigma(t)$ and when $n$ is even and odd. Our results obtained here extend and improve the results of Chen and Qu (J. Appl. Math. Comput. 44(1-2):357-377, 2014) and Zhang et al. (Appl. Math. Comput. 275:324-334, 2016).

MSC: 34K11; 34N05; 39A10; 39A13; 39A21; 39A99

Keywords: asymptotic behavior; oscillation; higher order; dynamic equations; dynamic inequality; time scales

\section{Introduction}

We are concerned with the asymptotic and oscillatory behavior of the higher-order nonlinear functional dynamic equation

$$
\begin{aligned}
& \left\{r_{n-1}(t) \phi_{\alpha_{n-1}}\left[\left(r_{n-2}(t)\left(\cdots\left(r_{1}(t) \phi_{\alpha_{1}}\left[x^{\Delta}(t)\right]\right)^{\Delta} \cdots\right)^{\Delta}\right)^{\Delta}\right]\right\}^{\Delta} \\
& \quad+\sum_{\nu=0}^{N} p_{\nu}(t) \phi_{\gamma_{v}}\left(x\left(g_{v}(t)\right)\right)=0
\end{aligned}
$$

on an above-unbounded time scale $\mathbb{T}$, assuming without loss of generality that $t_{0} \in \mathbb{T}$. For $A \subset \mathbb{T}$ and $B \subset \mathbb{R}$, we denote by $C_{\mathrm{rd}}(A, B)$ the space of right-dense continuous functions from $A$ to $B$ and by $C_{\mathrm{rd}}^{1}(A, B)$ the set of functions in $C_{\mathrm{rd}}(A, B)$ with right-dense continuous

(c) The Author(s) 2017. This article is distributed under the terms of the Creative Commons Attribution 4.0 International License (http://creativecommons.org/licenses/by/4.0/), which permits unrestricted use, distribution, and reproduction in any medium, provided you give appropriate credit to the original author(s) and the source, provide a link to the Creative Commons license, and indicate if changes were made. 
$\Delta$-derivatives. We refer the readers to the books by Bohner and Peterson $[3,4]$ for an excellent introduction of calculus of time scales. Throughout this paper, we suppose that:

(i) $n, N \in \mathbb{N}, n \geq 2$, and $\phi_{\beta}(u):=|u|^{\beta-1} u, \beta>0$;

(ii) $r_{i} \in C_{\mathrm{rd}}\left(\left[t_{0}, \infty\right)_{\mathbb{T}},(0, \infty)\right)$ for $i=1,2, \ldots, n-1$ are such that

$$
\int_{t_{0}}^{\infty} r_{i}^{-1 / \alpha_{i}}(\tau) \Delta \tau=\infty
$$

(iii) $\alpha_{i}>0, i=1,2, \ldots, n-1$, and $\gamma_{v}>0, v=0,1, \ldots, N$, are constants such that

$$
\gamma_{v}>\gamma_{0}, \quad v=1,2, \ldots, l \quad \text { and } \quad \gamma_{v}<\gamma_{0}, \quad v=l+1, l+2, \ldots, N
$$

(iv) $p_{v} \in C_{\mathrm{rd}}\left(\left[t_{0}, \infty\right)_{\mathbb{T}},[0, \infty)\right), v=0,1, \ldots, N$, are such that not all of the $p_{v}(t)$ vanish in a neighborhood of infinity;

(v) $g_{v}: \mathbb{T} \rightarrow \mathbb{T}$ are rd-continuous functions such that $\lim _{t \rightarrow \infty} g_{v}(t)=\infty, v=0,1, \ldots, N$.

By a solution of equation (1.1) we mean a function $x \in C_{\mathrm{rd}}^{1}\left(\left[T_{x}, \infty\right)_{\mathbb{T}}, \mathbb{R}\right)$ for some $T_{x} \geq 0$ such that $x^{[i]} \in C_{\mathrm{rd}}^{1}\left(\left[T_{x}, \infty\right)_{\mathbb{T}}, \mathbb{R}\right), i=1,2, \ldots, n-1$, that satisfies equation $(1.1)$ on $\left[T_{x}, \infty\right)_{\mathbb{T}}$, where

$$
x^{[i]}:=r_{i} \phi_{\alpha_{i}}\left[\left(x^{[i-1]}\right)^{\Delta}\right], \quad i=1,2, \ldots, n \text {, with } r_{n}=1, \alpha_{n}=1 \text {, and } x^{[0]}=x .
$$

A solution $x(t)$ of equation (1.1) is said to be oscillatory if it is neither eventually positive nor eventually negative. Otherwise, it is nonoscillatory.

Oscillation criteria for higher-order dynamic equations on time scales have been studied by many authors. For instance, Grace et al. [5] obtained sufficient conditions for oscillation for the higher-order nonlinear dynamic equation

$$
x^{\Delta^{n}}(t)+p(t)\left(x^{\sigma}(g(t))\right)^{\gamma}=0,
$$

where $\gamma$ is the quotient of positive odd integers, and where $g(t) \leq t$. In [5], some comparison criteria have been studied when $g(t) \leq t$, and some oscillation criteria are given when $n$ is even and $g(t)=t$. The results in [5] have been proved when

$$
\int_{t_{0}}^{\infty} \int_{t}^{\infty} \int_{s}^{\infty} p(u) \Delta u \Delta s \Delta t=\infty
$$

Wu et al. [6] established Kamanev-type oscillation criteria for the higher-order nonlinear dynamic equation

$$
\left\{r_{n-1}(t)\left[\left(r_{n-2}(t)\left(\cdots\left(r_{1}(t) x^{\Delta}(t)\right)^{\Delta} \cdots\right)^{\Delta}\right)^{\Delta}\right]^{\alpha}\right\}^{\Delta}+f(t, x(g(t)))=0,
$$

where $\alpha$ is the quotient of positive odd integers, $g: \mathbb{T} \rightarrow \mathbb{T}$ with $g(t)>t$ and $\lim _{t \rightarrow \infty} g(t)=$ $\infty$, and there exists a positive rd-continuous function $p(t)$ such that $\frac{f(t, u)}{u^{\alpha}} \geq p(t)$ for $u \neq 0$. Sun et al. [7] proved some criteria for oscillation and asymptotic behavior of the dynamic equation

$$
\left\{r_{n-1}(t)\left[\left(r_{n-2}(t)\left(\cdots\left(r_{1}(t) x^{\Delta}(t)\right)^{\Delta} \cdots\right)^{\Delta}\right)^{\Delta}\right]^{\alpha}\right\}^{\Delta}+f(t, x(g(t)))=0
$$


where $\alpha \geq 1$ is the quotient of positive odd integers, $g: \mathbb{T} \rightarrow \mathbb{T}$ is an increasing differentiable function with $g(t) \leq t, g \circ \sigma=\sigma \circ g$, and $\lim _{t \rightarrow \infty} g(t)=\infty$, and there exists a positive rd-continuous function $p(t)$ such that $\frac{f(t, u)}{u^{\beta}} \geq p(t)$ for $u \neq 0$ and $\beta \geq 1$ is the quotient of positive odd integers. Sun et al. [8] studied quasilinear dynamic equations of the form

$$
\left\{r_{n-1}(t)\left[\left(r_{n-2}(t)\left(\cdots\left(r_{1}(t) x^{\Delta}(t)\right)^{\Delta} \cdots\right)^{\Delta}\right)^{\Delta}\right]^{\alpha}\right\}^{\Delta}+p(t) x^{\beta}(t)=0,
$$

where $\alpha, \beta$ are the quotients of positive odd integers. Also, the results obtained in [6-8] are presented when

$$
\int_{t_{0}}^{\infty} \frac{1}{r_{n-2}(t)}\left\{\int_{t}^{\infty}\left[\frac{1}{r_{n-1}(s)} \int_{s}^{\infty} p(u) \Delta u\right]^{1 / \alpha} \Delta s\right\} \Delta t=\infty
$$

Hassan and Kong [9] obtained asymptotics and oscillation criteria for the $n$ th-order halflinear dynamic equation

$$
\left(x^{[n-1]}\right)^{\Delta}(t)+p(t) \phi_{\alpha[1, n-1]}(x(g(t)))=0,
$$

where $\alpha[1, n-1]:=\alpha_{1} \cdots \alpha_{n-1}$, and Grace and Hassan [10] further studied the asymptotics and oscillation for the higher-order nonlinear dynamic equation

$$
\left(x^{[n-1]}\right)^{\Delta}(t)+p(t) \phi_{\gamma}\left(x^{\sigma}(g(t))\right)=0 .
$$

However, the establishment of the results in [10] requires the restriction on the time scale $\mathbb{T}$ that $g^{*} \circ \sigma=\sigma \circ g^{*}$ with $g^{*}(t)=\min \{t, g(t)\}$, which is hardly satisfied. Hassan [11] improved the results in $[9,10]$ and established oscillation criteria for the higher-order quasilinear dynamic equation

$$
\left(x^{[n-1]}\right)^{\Delta}(t)+p(t) \phi_{\gamma}(x(g(t)))=0
$$

when $n$ is even or odd and when $\alpha>\gamma, \alpha=\gamma$, and $\alpha<\gamma$ with $\alpha=\alpha_{1} \cdots \alpha_{n-1}$. Chen and Qu [1] considered the even-order advanced type dynamic equation with mixed nonlinearities

$$
\left\{r(t) \phi_{\gamma_{0}}\left(x^{\Delta^{n-1}}(t)\right)\right\}^{\Delta}+\sum_{\nu=0}^{N} p_{\nu}(t) \phi_{\gamma_{\nu}}\left(x\left(g_{\nu}(t)\right)\right)=0
$$

where $n \geq 2$ is even, $\gamma_{v}>0, g_{v}(t) \geq t$, and $\gamma_{1}>\cdots>\gamma_{l}>\gamma_{0}>\gamma_{l+1}>\cdots>\gamma_{N}>0$. Zhang etal. [2] studied the dynamic equation (1.7), where $n \geq 2$ is integer and $g_{v}^{\Delta}(t)>0$, and obtained some of the results in [2] when $\gamma_{0} \geq 1$. Also, the results obtained in $[1,2]$ are given when

$$
\int_{t_{0}}^{\infty}\left[\int_{v}^{\infty}\left(r^{-1}(s) \int_{s}^{\infty} \sum_{v=0}^{N} p_{v}(\tau) \Delta \tau\right)^{1 / \gamma_{0}} \Delta s\right] \Delta v=\infty .
$$

Huang [12] extended the work in [1] to the neutral advanced dynamic equation

$$
\left\{r(t) \phi_{\alpha}\left(y^{\Delta^{n-1}}(t)\right)\right\}^{\Delta}+\sum_{\nu=0}^{N} p_{\nu}(t) \phi_{\gamma_{v}}\left(x\left(g_{\nu}(t)\right)\right)=0,
$$


where $n \geq 2$ is integer, $y(t):=x(t)+p(t) x(g(t)), \gamma_{v}>0, g(t) \leq t$, and $g_{v}(t) \geq t$. For more results on dynamic equations, we refer the reader to the papers [13-29].

In this paper, we will discuss the higher-order nonlinear dynamic equation (1.1) with mixed nonlinearities on a general time scale without any restrictions on $g(t)$ and $\sigma(t)$ and also without conditions (1.5), (1.6), and (1.8). The results in this paper improve the results in $[1,2,5-10]$ on the oscillation of various dynamic equations.

\section{Main results}

We introduce the following notations:

$$
k_{+}:=\max \{k, 0\}, \quad k_{-}:=\max \{-k, 0\} \quad \text { for any } k \in \mathbb{R},
$$

and

$$
\alpha[h, k]:= \begin{cases}\alpha_{h} \cdots \alpha_{k}, & h \leq k \\ 1, & h>k\end{cases}
$$

with $\alpha=\gamma_{0}=\alpha[1, n-1]$ and $\beta_{i}=\alpha[1, i]$. For any $t, s \in \mathbb{T}$ and for a fixed $m \in\{0,1, \ldots, n-1\}$, define the functions $R_{m, j}(t, s), j=0,1, \ldots, m$, and $\hat{p}_{j}(t), j=0,1, \ldots, n-1$, by the following recurrence formulas:

$$
R_{m, j}(t, s):= \begin{cases}1, & j=0, \\ \int_{s}^{t}\left[\frac{R_{m, j-1}(\tau, s)}{r_{m-j+1}(\tau)}\right]^{1 / \alpha_{m-j+1}} \Delta \tau, & j=1,2, \ldots, m,\end{cases}
$$

and

$$
\hat{p}_{j}(t):= \begin{cases}\sum_{\nu=0}^{N} p_{v}(t), & j=0, \\ {\left[\frac{1}{r_{n-j}(t)} \int_{t}^{\infty} \hat{p}_{j-1}(\tau) \Delta \tau\right]^{1 / \alpha_{n-j}},} & j=1,2, \ldots, n-1 .\end{cases}
$$

For a fixed $m \in\{0, \ldots, n-1\}$, define the functions $\bar{p}_{m, j}(t, s), j=0,1,2, \ldots, n-1$, by the recurrence formula

$$
\bar{p}_{m, j}(t, s):= \begin{cases}p_{m}(t, s), & j=0, \\ {\left[\frac{1}{r_{n-j}(t)} \int_{t}^{\infty} \bar{p}_{m, j-1}(\tau, s) \Delta \tau\right]^{1 / \alpha_{n-j}},} & j=1,2, \ldots, n-1,\end{cases}
$$

with

$$
\varphi_{m, v}\left(t, t_{1}\right):= \begin{cases}1, & g_{\nu}(t) \geq \sigma(t), \\ \frac{R_{m, m}\left(g_{v}(t), t_{1}\right)}{R_{m, m}\left(\sigma(t), t_{1}\right)}, & g_{\nu}(t) \leq \sigma(t),\end{cases}
$$

and

$$
p_{m}(t, s)=p_{0}(t) \phi_{\alpha}\left(\varphi_{m, 0}(t, s)\right)+\prod_{\nu=1}^{N}\left[\frac{p_{v}(t) \phi_{\gamma_{v}}\left(\varphi_{m, v}(t, s)\right)}{\eta_{v}}\right]^{\eta_{v}}
$$

such that

$$
\sum_{\nu=1}^{N} \gamma_{\nu} \eta_{\nu}=\alpha \quad \text { and } \quad \sum_{\nu=1}^{N} \eta_{\nu}=1
$$


where

$$
\delta(t, s):= \begin{cases}{\left[\int_{t}^{\infty} \bar{p}_{m, n-m-1}(\tau, s) \Delta \tau\right]^{1 / \beta_{m}-1},} & 0<\beta_{m} \leq 1, \\ R_{m, m}^{\beta_{m}-1}(t, s), & \beta_{m} \geq 1,\end{cases}
$$

provided that the improper integrals involved are convergent.

In the sequel, we present conditions that guarantee the following conclusions:

(C) (i) every solution of equation (1.1) is oscillatory if $n$ is even;

(ii) every solution of equation (1.1) either is oscillatory or tends to zero eventually if $n$ is odd.

Theorem 2.1 Let conditions (i)-(v) hold. Furthermore, for each $i \in\{1,2, \ldots, n-1\}$ and sufficiently large $T, T_{1} \in\left[t_{0}, \infty\right)_{\mathbb{T}}$, one of the following conditions is satisfied:

(a) either $\int_{T}^{\infty} \bar{p}_{i, n-i-1}\left(\tau, T_{1}\right) \Delta \tau=\infty$, or $\int_{T}^{\infty} \bar{p}_{i, n-i-1}\left(\tau, T_{1}\right) \Delta \tau<\infty$ and either

$$
\limsup _{t \rightarrow \infty} R_{i, i}^{\beta_{i}}\left(t, T_{1}\right) \int_{t}^{\infty} \bar{p}_{i, n-i-1}\left(\tau, T_{1}\right) \Delta \tau>1
$$

or

$$
\limsup _{t \rightarrow \infty} R_{i, i}\left(t, T_{1}\right)\left(\int_{t}^{\infty} \bar{p}_{i, n-i-1}\left(\tau, T_{1}\right) \Delta \tau\right)^{1 / \beta_{i}}>1
$$

(b) there exists $\rho_{i} \in C_{\mathrm{rd}}^{1}\left(\left[t_{0}, \infty\right)_{\mathbb{T}},(0, \infty)\right)$ such that

$$
\limsup _{t \rightarrow \infty} \int_{T}^{t}\left[\rho_{i}(\tau) \bar{p}_{i, n-i-1}\left(\tau, T_{1}\right)-\frac{\left(\rho_{i}^{\Delta}(\tau)\right)_{+}}{R_{i, i}^{\beta_{i}}\left(\sigma(\tau), T_{1}\right)}\right] \Delta \tau=\infty ;
$$

(c) there exists $\rho_{i} \in C_{\mathrm{rd}}^{1}\left(\left[t_{0}, \infty\right)_{\mathbb{T}},(0, \infty)\right)$ such that

$$
\begin{aligned}
\limsup _{t \rightarrow \infty} \int_{T}^{t}\left[\rho_{i}(\tau) \bar{p}_{i, n-i-1}\left(\tau, T_{1}\right)\right. \\
\left.-\frac{1}{\rho_{i}^{\beta_{i}}(\tau)}\left[\frac{\left(\rho_{i}^{\Delta}(\tau)\right)_{+}}{1+\beta_{i}}\right]^{1+\beta_{i}}\left[\frac{r_{1}(\tau)}{R_{i, i-1}\left(\tau, T_{1}\right)}\right]^{\beta_{i} / \alpha_{1}}\right] \Delta \tau=\infty ;
\end{aligned}
$$

(d) there exist $\rho_{i} \in C_{\mathrm{rd}}^{1}\left(\left[t_{0}, \infty\right)_{\mathbb{T}},(0, \infty)\right)$ and $H_{i}, h_{i} \in C_{\mathrm{rd}}(\mathbb{D}, \mathbb{R})$, where $\mathbb{D} \equiv\left\{(t, \tau): t \geq \tau \geq t_{0}\right\}$, such that

$$
H_{i}(t, t)=0, \quad t \geq t_{0}, \quad H_{i}(t, \tau)>0, \quad t>\tau \geq t_{0},
$$

and $H_{i}$ has a nonpositive continuous $\Delta$-partial derivative $H_{i}^{\Delta_{\tau}}(t, \tau)$ with respect to the second variable and satisfies

$$
H_{i}^{\Delta_{\tau}}(t, \tau)+H_{i}(t, \tau) \frac{\rho_{i}^{\Delta}(\tau)}{\rho_{i}^{\sigma}(\tau)}=-\frac{h_{i}(t, \tau)}{\rho_{i}^{\sigma}(\tau)} H_{i}^{\beta_{i} /\left(1+\beta_{i}\right)}(t, \tau)
$$


and

$$
\begin{aligned}
\limsup _{t \rightarrow \infty} & \frac{1}{H_{i}(t, T)} \int_{T}^{t}\left[\rho_{i}(\tau) \bar{p}_{i, n-i-1}\left(\tau, T_{1}\right) H_{i}(t, \tau)\right. \\
- & \left.\frac{1}{\rho_{i}^{\beta_{i}}(\tau)}\left[\frac{\left(h_{i}(t, \tau)\right)_{-}}{1+\beta_{i}}\right]^{1+\beta_{i}}\left[\frac{r_{1}(\tau)}{R_{i, i-1}\left(\tau, T_{1}\right)}\right]^{\beta_{i} / \alpha_{1}}\right] \Delta \tau=\infty
\end{aligned}
$$

(e) there exists $\rho_{i} \in C_{\mathrm{rd}}^{1}\left(\left[t_{0}, \infty\right)_{\mathbb{T}},(0, \infty)\right)$ such that

$$
\begin{aligned}
& \underset{t \rightarrow \infty}{\limsup } \int_{T}^{t}\left[\rho_{i}(\tau) \bar{p}_{i, n-i-1}\left(\tau, T_{1}\right)\right. \\
& \left.\quad-\frac{\left(\rho_{i}^{\Delta}(\tau)\right)^{2}}{4 \beta_{i} \rho_{i}(\tau) \delta^{\sigma}\left(\tau, T_{1}\right)}\left[\frac{r_{1}(\tau)}{R_{i, i-1}\left(\tau, T_{1}\right)}\right]^{1 / \alpha_{1}}\right] \Delta \tau=\infty
\end{aligned}
$$

(f) there exist $\rho_{i} \in C_{\mathrm{rd}}^{1}\left(\left[t_{0}, \infty\right)_{\mathbb{T}},(0, \infty)\right)$ and $H_{i}, h_{i} \in C_{\mathrm{rd}}(\mathbb{D}, \mathbb{R})$, where $\mathbb{D} \equiv\left\{(t, \tau): t \geq \tau \geq t_{0}\right\}$, such that (2.7) holds and $H_{i}$ has a nonpositive continuous $\Delta$-partial derivative $H_{i}^{\Delta_{\tau}}(t, \tau)$ with respect to the second variable and satisfies

$$
H_{i}^{\Delta_{\tau}}(t, \tau)+H_{i}(t, \tau) \frac{\rho_{i}^{\Delta}(\tau)}{\rho_{i}^{\sigma}(\tau)}=-\frac{h_{i}(t, \tau)}{\rho_{i}^{\sigma}(\tau)} \sqrt{H_{i}(t, \tau)}
$$

and

$$
\begin{aligned}
& \limsup _{t \rightarrow \infty} \frac{1}{H_{i}(t, T)} \int_{T}^{t}\left[\rho_{i}(\tau) \bar{p}_{i, n-i-1}\left(\tau, T_{1}\right) H_{i}(t, \tau)\right. \\
& \left.\quad-\frac{\left[\left(h_{i}(t, \tau)\right)_{-}\right]^{2}}{4 \beta_{i} \rho_{i}(\tau) \delta^{\sigma}\left(\tau, T_{1}\right)}\left[\frac{r_{1}(\tau)}{R_{i, i-1}\left(\tau, T_{1}\right)}\right]^{1 / \alpha_{1}}\right] \Delta \tau=\infty
\end{aligned}
$$

Moreover, for the case where $n$ is odd, assume that, for an integer $j \in\{0,1, \ldots, n-1\}$,

$$
\int_{T}^{\infty} \hat{p}_{j}(\tau) \Delta \tau=\infty
$$

Then conclusions (C) hold.

Example 2.1 Consider the higher-order nonlinear dynamic equation (1.1), where $\beta_{i}=$ $\alpha[1, i] \leq 1$ and $r_{1}(t):=\frac{t^{\xi}}{\beta_{1}}$ with

$$
\xi= \begin{cases}>0 & \text { if } n \text { is even } \\ \leq 0 & \text { if } n \text { is odd }\end{cases}
$$

and where

$$
r_{i}(t):=\frac{t^{\alpha_{i}}}{\beta_{i}}, \quad i=2, \ldots, n-1 \quad \text { and } \quad p_{0}(t):=\frac{\zeta}{t^{\alpha+1} \phi_{\alpha}\left(\varphi_{i, 0}\left(t, t_{0}\right)\right)} \quad \text { with } \zeta>0 .
$$

Choose an $n$-tuple $\left(\eta_{1}, \eta_{2}, \ldots, \eta_{n}\right)$ with $0<\eta_{j}<1$ satisfying (2.4). It is clear that conditions (1.2) hold since

$$
\int_{t_{0}}^{\infty} r_{1}^{-1 / \alpha_{1}}(\tau) \Delta \tau=\beta_{1}^{1 / \beta_{1}} \int_{t_{0}}^{\infty} \frac{\Delta \tau}{\tau^{\xi / \alpha_{1}}}=\infty \quad \text { and } \quad \int_{t_{0}}^{\infty} r_{i}^{-1 / \alpha_{i}}(\tau) \Delta \tau=\beta_{i}^{1 / \alpha_{i}} \int_{t_{0}}^{\infty} \frac{\Delta \tau}{\tau}=\infty
$$


and hence (2.6) holds. Also,

$$
\begin{aligned}
\hat{p}_{n-1}(t) & =\left[\frac{1}{r_{1}(t)} \int_{t}^{\infty} \hat{p}_{n-2}(\tau) \Delta \tau\right]^{1 / \alpha_{1}} \\
& \geq \zeta^{1 / \alpha}\left[\frac{\alpha_{1}}{t^{\xi}} \int_{t}^{\infty} \frac{1}{\tau^{\alpha_{1}+1}} \Delta \tau\right]^{1 / \alpha_{1}} \\
& \geq \zeta^{1 / \alpha}\left[\frac{1}{t^{\xi}} \int_{t}^{\infty}\left(\frac{-1}{\tau^{\alpha_{1}}}\right)^{\Delta} \Delta \tau\right]^{1 / \alpha_{1}}=\frac{\zeta^{1 / \alpha}}{t^{1+\xi / \alpha_{1}}}
\end{aligned}
$$

If $n$ is odd, then

$$
\int_{T}^{\infty} \hat{p}_{n-1}(\tau) \Delta \tau=\zeta^{1 / \alpha} \int_{T}^{\infty} \frac{\Delta \tau}{\tau^{1+\xi / \alpha_{1}}}=\infty
$$

so that condition (2.13) holds. Then, by Theorem 2.1(c) conclusions (C) hold if

$$
\zeta^{1 / \alpha[i+1, n-1]}>\left[\frac{1}{\alpha_{1}}\right]^{\beta_{i} / \alpha_{1}}\left[\frac{\beta_{i}}{1+\beta_{i}}\right]^{1+\beta_{i}}
$$

\section{Lemmas}

In order to prove the main results, we need the following lemmas. The first two lemmas are extensions of Lemmas 1 and 2 in [9] to the nonlinear equation (1.1) with exactly the same proof.

Lemma 3.1 Let $x(t) \in C_{\mathrm{rd}}^{n}(\mathbb{T},[0, \infty))$. Assume that $\left(x^{[n-1]}\right)^{\Delta}(t)$ is of eventually one sign and not identically zero. Then there exists an integer $m \in\{0,1, \ldots, n-1\}$ with $m+n$ odd for $\left(x^{[n-1]}\right)^{\Delta}(t) \leq 0$ or with $m+n$ even for $\left(x^{[n-1]}\right)^{\Delta}(t) \geq 0$ such that

$$
x^{[k]}(t)>0 \quad \text { for } k=0,1, \ldots, m
$$

and

$$
(-1)^{m+k} x^{[k]}(t)>0 \quad \text { for } k=m, m+1, \ldots, n-1
$$

eventually.

Lemma 3.2 Assume that equation (1.1) has an eventually positive solution $x(t)$ and $m \in$ $\{0,1, \ldots, n-1\}$ is given in Lemma 3.1 such that (3.1) and (3.2) hold for $t \in\left[t_{1}, \infty\right)_{\mathbb{T}}$ for some $t_{1} \in\left[t_{0}, \infty\right)_{\mathbb{T}}$. Then the following hold for $t \in\left(t_{1}, \infty\right)_{\mathbb{T}}$ :

(a) for $i=0,1, \ldots, m$,

$$
\frac{x^{[m-i]}(t)}{R_{m, i}\left(t, t_{1}\right)} \quad \text { is strictly decreasing; }
$$

(b) for $i \in\{0,1, \ldots, m\}$ and $j=0,1, \ldots, m-i$,

$$
x^{[j]}(t) \geq \phi_{\alpha[j+1, m-i]}^{-1}\left[\frac{x^{[m-i]}(t)}{R_{m, i}\left(t, t_{1}\right)}\right] R_{m, m-j}\left(t, t_{1}\right) .
$$


Lemma 3.3 Assume that equation (1.1) has an eventually positive solution $x(t)$ and $m$ is given in Lemma 3.1 such that $m \in\{1,2, \ldots, n-1\}$ and (3.1) and (3.2) hold for $t \geq t_{1} \in$ $\left[t_{0}, \infty\right)_{\mathbb{T}}$. Then, for $t \in\left[t_{2}, \infty\right)_{\mathbb{T}}$, where $g_{v}(t)>t_{1}$ for $t \geq t_{2}$, and for $j=m, m+1, \ldots, n-1$,

$$
\int_{t}^{\infty} \bar{p}_{m, n-j-1}\left(\tau, t_{1}\right) \Delta \tau<\infty
$$

and

$$
(-1)^{m+j} x^{[j]}(t) \geq \phi_{\alpha[1, j]}\left(x^{\sigma}(t)\right) \int_{t}^{\infty} \bar{p}_{m, n-j-1}\left(\tau, t_{1}\right) \Delta \tau .
$$

Proof We show it by a backward induction. By Lemma 3.1 with $m \geq 1$ we see that $x(t)$ is strictly increasing on $\left[t_{1}, \infty\right)_{\mathbb{T}}$. As a result, (3.1) and (3.2) hold for $t \in\left[t_{1}, \infty\right)_{\mathbb{T}}$. Let $t \in\left[t_{1}, \infty\right)_{\mathbb{T}}$ be fixed. Then, for $v=0,1, \ldots, N$, if $g_{v}(t) \geq \sigma(t)$, then $x\left(g_{v}(t)\right) \geq x(t)$ by the fact that $x(t)$ is strictly increasing. Now consider the case where $g_{v}(t) \leq \sigma(t)$. In view of Lemma 3.2(a), we see that for $i=m, \frac{x(t)}{R_{m, m}\left(t, t_{1}\right)}$ is decreasing on $\left(t_{1}, \infty\right)_{\mathbb{T}}$ and that there exists $t_{2} \geq t_{1}$ such that $g_{v}(t)>t_{1}$ for $t \geq t_{2}$, so that

$$
x\left(g_{v}(t)\right) \geq \frac{R_{m, m}\left(g_{v}(t), t_{1}\right)}{R_{m, m}\left(\sigma(t), t_{1}\right)} x^{\sigma}(t) \quad \text { for } t \in\left[t_{2}, \infty\right)_{\mathbb{T}} .
$$

In both cases, we have

$$
x\left(g_{v}(t)\right) \geq \varphi_{m, v}\left(t, t_{1}\right) x^{\sigma}(t) \quad \text { for } t \in\left[t_{2}, \infty\right)_{\mathbb{T}} .
$$

Therefore,

$$
\begin{aligned}
\sum_{\nu=0}^{N} p_{\nu}(t) \phi_{\gamma_{v}}\left(x\left(g_{v}(t)\right)\right) & \geq \sum_{\nu=0}^{N} p_{v}(t) \phi_{\gamma_{v}}\left(\varphi_{m, v}\left(t, t_{1}\right)\right)\left[x^{\sigma}(t)\right]^{\gamma_{\nu}} \\
& =\phi_{\alpha}\left(x^{\sigma}(t)\right) \sum_{\nu=0}^{N} p_{\nu}(t) \phi_{\gamma_{v}}\left(\varphi_{m, v}\left(t, t_{1}\right)\right)\left[x^{\sigma}(t)\right]^{\gamma_{\nu}-\alpha}
\end{aligned}
$$

Using the arithmetic-geometric mean inequality (see [30], p.17), we have

$$
\sum_{\nu=1}^{N} \eta_{\nu} v_{v} \geq \prod_{v=1}^{N} v_{v}^{\eta_{v}} \quad \text { for any } v_{v} \geq 0, v=1, \ldots, N .
$$

Then, for $t \geq T_{1}$,

$$
\begin{aligned}
& \sum_{\nu=0}^{N} p_{\nu}(t) \phi_{\gamma_{v}}\left(\varphi_{m, v}\left(t, t_{1}\right)\right)\left[x^{\sigma}(t)\right]^{\gamma_{v}-\alpha} \\
& \quad=p_{0}(t) \phi_{\alpha}\left(\varphi_{m, 0}\left(t, t_{1}\right)\right)+\sum_{\nu=1}^{N} \eta_{\nu} \frac{p_{v}(t) \phi_{\gamma_{v}}\left(\varphi_{m, v}\left(t, t_{1}\right)\right)}{\eta_{\nu}}\left[x^{\sigma}(t)\right]^{\gamma_{v}-\alpha} \\
& \quad \geq p_{0}(t) \phi_{\alpha}\left(\varphi_{m, 0}\left(t, t_{1}\right)\right)+\prod_{\nu=1}^{N}\left[\frac{p_{\nu}(t) \phi_{\gamma_{v}}\left(\varphi_{m, v}\left(t, t_{1}\right)\right)}{\eta_{v}}\right]^{\eta_{\nu}}\left[x^{\sigma}(t)\right]^{\eta_{\nu}\left(\gamma_{v}-\alpha\right)}
\end{aligned}
$$


In view of (2.4), we have

$$
\sum_{\nu=1}^{N} \gamma_{\nu} \eta_{\nu}-\alpha \sum_{\nu=1}^{N} \eta_{\nu}=0
$$

Hence,

$$
\begin{aligned}
& \sum_{v=0}^{N} p_{v}(t) \phi_{\gamma_{v}}\left(\varphi_{m, v}\left(t, t_{1}\right)\right)\left[x^{\sigma}(t)\right]^{\gamma_{v}-\alpha} \\
& \quad \geq p_{0}(t) \phi_{\alpha}\left(\varphi_{m, 0}\left(t, t_{1}\right)\right)+\prod_{v=1}^{N}\left[\frac{p_{v}(t) \phi_{\gamma_{v}}\left(\varphi_{m, v}\left(t, t_{1}\right)\right)}{\eta_{v}}\right]^{\eta_{v}}=p\left(t, t_{1}\right) .
\end{aligned}
$$

This, together with (1.1), shows that, for $t \in\left[t_{2}, \infty\right)_{\mathbb{T}}$,

$$
-\left(x^{[n-1]}(t)\right)^{\Delta} \geq p\left(t, t_{1}\right) \phi_{\alpha}\left(x^{\sigma}(t)\right)=\bar{p}_{m, 0}\left(t, t_{1}\right) \phi_{\alpha}\left(x^{\sigma}(t)\right) .
$$

Replacing $t$ by $\tau$ in (3.6), integrating from $t \in\left[t_{2}, \infty\right)_{\mathbb{T}}$ to $v \in[t, \infty)_{\mathbb{T}}$, and using (3.2), we have

$$
\begin{aligned}
x^{[n-1]}(t) & >-x^{[n-1]}(v)+x^{[n-1]}(t) \geq \int_{t}^{v} \bar{p}_{m, 0}\left(\tau, t_{1}\right) \phi_{\alpha}\left(x^{\sigma}(\tau)\right) \Delta \tau \\
& \geq \phi_{\alpha}\left(x^{\sigma}(t)\right) \int_{t}^{v} \bar{p}_{m, 0}\left(\tau, t_{1}\right) \Delta \tau .
\end{aligned}
$$

Hence, by taking limits as $v \rightarrow \infty$ we obtain that

$$
x^{[n-1]}(t) \geq \phi_{\alpha}\left(x^{\sigma}(t)\right) \int_{t}^{\infty} \bar{p}_{m, 0}\left(\tau, t_{1}\right) \Delta \tau
$$

This shows that $\int_{t}^{\infty} \bar{p}_{m, 0}\left(\tau, t_{1}\right) \Delta \tau<\infty$ and (3.5) holds for $j=n-1$. Assume that $\int_{t}^{\infty} \bar{p}_{m, n-j-1}\left(\tau, t_{1}\right) \Delta \tau<\infty$ and (3.5) holds for some $j \in\{m+1, m+2, \ldots, n-1\}$. Then, for (3.5),

$$
\begin{aligned}
(-1)^{m+j}\left[x^{[j-1]}(t)\right]^{\Delta} & =(-1)^{m+j} \phi_{\alpha_{j}}^{-1}\left[\frac{x^{[j]}(t)}{r_{j}(t)}\right] \\
& \geq \phi_{\alpha_{j}}^{-1}\left\{\phi_{\alpha[1, j]}\left(x^{\sigma}(t)\right)\right\}\left[\frac{1}{r_{j}(t)} \int_{t}^{\infty} \bar{p}_{m, n-j-1}\left(\tau, t_{1}\right) \Delta \tau\right]^{1 / \alpha_{j}} \\
& =\phi_{\alpha[1, j-1]}\left(x^{\sigma}(t)\right) \bar{p}_{m, n-j}\left(t, t_{1}\right) .
\end{aligned}
$$

Replacing $t$ by $\tau$ and then integrating it from $t \in\left[t_{2}, \infty\right)_{\mathbb{T}}$ to $v \in[t, \infty)_{\mathbb{T}}$, we have

$$
\begin{aligned}
(-1)^{m+j-1} x^{[j-1]}(t) & >(-1)^{m+j}\left(x^{[j-1]}(v)-x^{[j-1]}(t)\right) \\
& \geq \int_{t}^{v} \phi_{\alpha[1, j-1]}\left(x^{\sigma}(\tau)\right) \bar{p}_{m, n-j}\left(\tau, t_{1}\right) \Delta \tau \\
& \geq \phi_{\alpha[1, j-1]}\left(x^{\sigma}(t)\right) \int_{t}^{v} \bar{p}_{m, n-j}\left(\tau, t_{1}\right) \Delta \tau .
\end{aligned}
$$


Taking limits as $v \rightarrow \infty$, we obtain that

$$
(-1)^{m+j-1} x^{[j-1]}(t) \geq \phi_{\alpha[1, j-1]}\left(x^{\sigma}(t)\right) \int_{t}^{\infty} \bar{p}_{m, n-j}\left(\tau, t_{1}\right) \Delta \tau .
$$

This shows that $\int_{t}^{\infty} \bar{p}_{m, n-j}\left(\tau, t_{1}\right) \Delta \tau<\infty$ and (3.5) holds for $j-1$. Therefore, the conclusion holds.

The following lemma improves [31], Lemma 1; also see [32-34].

Lemma 3.4 Let (1.3) hold. Then, there exists an $N$-tuple $\left(\eta_{1}, \eta_{2}, \ldots, \eta_{N}\right)$ with $\eta_{v}>0$ satisfying (2.4).

Lemma 3.5 (see [35]) Let $\omega(u)=a u-b u^{1+1 / \beta}$, where $a, u \geq 0$ and $b, \beta>0$. Then

$$
\omega(u) \leq\left(\frac{\beta}{b}\right)^{\beta}\left(\frac{a}{1+\beta}\right)^{1+\beta} .
$$

\section{Proofs of main results}

Proof of Theorem 2.1 Assume that equation (1.1) has a nonoscillatory solution $x(t)$. Then, without loss of generality, assume that $x(t)>0$ and $x\left(g_{v}(t)\right)>0$ for $t \in\left[t_{0}, \infty\right)_{\mathbb{T}}$. It follows from Lemma 3.1 that there exists an integer $m \in\{0,1, \ldots, n-1\}$ with $m+n$ odd such that (3.1) and (3.2) hold for $t \in\left[t_{1}, \infty\right)_{\mathbb{T}}$ for some $t_{1} \in\left[t_{0}, \infty\right)_{\mathbb{T}}$. Let $t_{2} \geq t_{1}$ be such that $g_{v}(t)>t_{1}$ for $t \in\left[t_{2}, \infty\right)_{\mathbb{T}}$.

(i) Assume that $m \geq 1$.

Part I: Assume that (a) holds. By Lemma 3.3 we have that, for $j=m$,

$$
\int_{t}^{\infty} \bar{p}_{m, n-m-1}\left(\tau, t_{1}\right) \Delta \tau<\infty
$$

which contradicts $\int_{t}^{\infty} \bar{p}_{m, n-m-1}\left(\tau, t_{1}\right) \Delta \tau=\infty$. If $\int_{t}^{\infty} \bar{p}_{m, n-m-1}\left(\tau, t_{1}\right) \Delta \tau<\infty$, then by Lemma 3.3 we have that, for $j=m$,

$$
\begin{aligned}
x^{[m]}(t) & \geq \phi_{\alpha[1, m]}\left(x^{\sigma}(t)\right) \int_{t}^{\infty} \bar{p}_{m, n-m-1}\left(\tau, t_{1}\right) \Delta \tau \\
& \geq \phi_{\beta_{m}}(x(t)) \int_{t}^{\infty} \bar{p}_{m, n-m-1}\left(\tau, t_{1}\right) \Delta \tau .
\end{aligned}
$$

By Lemma 3.2(b) with $i=0$ and $j=0$ we get

$$
\begin{aligned}
x(t) & \geq \phi_{\alpha[1, m]}^{-1}\left(x^{[m]}(t)\right) R_{m, m}\left(t, t_{1}\right) \\
& =\phi_{\beta_{m}}^{-1}\left(x^{[m]}(t)\right) R_{m, m}\left(t, t_{1}\right) .
\end{aligned}
$$

Substituting (4.2) into (4.1), we obtain that

$$
1 \geq R_{m, m}^{\beta_{m}}\left(t, t_{1}\right) \int_{t}^{\infty} \bar{p}_{m, n-m-1}\left(\tau, t_{1}\right) \Delta \tau,
$$


which contradicts $\lim \sup _{t \rightarrow \infty} R_{m, m}^{\beta_{m}}\left(t, t_{1}\right) \int_{t}^{\infty} \bar{p}_{m, n-m-1}\left(\tau, t_{1}\right) \Delta \tau>1$. Substituting (4.1) into (4.2), we obtain that

$$
1 \geq R_{m, m}\left(t, t_{1}\right)\left(\int_{t}^{\infty} \bar{p}_{m, n-m-1}\left(\tau, t_{1}\right) \Delta \tau\right)^{1 / \beta_{m}}
$$

which contradicts $\lim \sup _{t \rightarrow \infty} R_{m, m}\left(t, t_{1}\right)\left(\int_{t}^{\infty} \bar{p}_{m, n-m-1}\left(\tau, t_{1}\right) \Delta \tau\right)^{1 / \beta_{m}}>1$.

Part II: Assume that (b) holds. Define

$$
w_{m}(t):=\rho_{m}(t) \frac{x^{[m]}(t)}{x^{\beta_{m}}(t)}
$$

By the product rule and the quotient rule we have

$$
\begin{aligned}
w_{m}^{\Delta}(t) & =\rho_{m}(t)\left(\frac{x^{[m]}(t)}{x^{\beta_{m}}(t)}\right)^{\Delta}+\rho_{m}^{\Delta}(t)\left(\frac{x^{[m]}(t)}{x^{\beta_{m}}(t)}\right)^{\sigma} \\
& =\rho_{m}(t)\left(\frac{x^{\beta_{m}}(t)\left(x^{[m]}(t)\right)^{\Delta}-\left(x^{\beta_{m}}(t)\right)^{\Delta} x^{[m]}(t)}{\left(x^{\beta_{m}}(t)\right)^{\sigma} x^{\beta_{m}}(t)}\right)+\rho_{m}^{\Delta}(t)\left(\frac{x^{[m]}(t)}{x^{\beta_{m}}(t)}\right)^{\sigma} \\
& =\rho_{m}(t) \frac{\left(x^{[m]}(t)\right)^{\Delta}}{\left(x^{\beta_{m}}(t)\right)^{\sigma}}-\rho_{m}(t) \frac{\left(x^{\beta_{m}}(t)\right)^{\Delta}}{\left(x^{\beta_{m}}(t)\right)^{\sigma}} \frac{x^{[m]}(t)}{x^{\beta_{m}}(t)}+\rho_{m}^{\Delta}(t)\left(\frac{x^{[m]}(t)}{x^{\beta_{m}}(t)}\right)^{\sigma} .
\end{aligned}
$$

From Lemma 3.3 with $j=m+1$ we have

$$
-x^{[m+1]}(t) \geq \phi_{\alpha[1, m+1]}\left(x^{\sigma}(t)\right) \int_{t}^{\infty} \bar{p}_{m, n-m-2}\left(\tau, t_{1}\right) \Delta \tau,
$$

which, together with (2.3), implies that, for $t \in\left[t_{1}, \infty\right)_{\mathbb{T}}$,

$$
\begin{aligned}
-\left(x^{[m]}(t)\right)^{\Delta} & \geq \phi_{\alpha[1, m]}\left(x^{\sigma}(t)\right)\left[\frac{1}{r_{m+1}(t)} \int_{t}^{\infty} \bar{p}_{m, n-m-2}\left(\tau, t_{1}\right) \Delta \tau\right]^{1 / \alpha_{m+1}} \\
& =\phi_{\beta_{m}}\left(x^{\sigma}(t)\right) \bar{p}_{m, n-m-1}\left(t, t_{1}\right) .
\end{aligned}
$$

Substituting (4.6) into (4.4), we obtain

$$
w_{m}^{\Delta}(t) \leq-\rho_{m}(t) \bar{p}_{m, n-m-1}\left(t, t_{1}\right)+\rho_{m}^{\Delta}(t)\left(\frac{x^{[m]}(t)}{x^{\beta_{m}}(t)}\right)^{\sigma}-\rho_{m}(t) \frac{\left(x^{\beta_{m}}(t)\right)^{\Delta}}{\left(x^{\beta_{m}}(t)\right)^{\sigma}} \frac{x^{[m]}(t)}{x^{\beta_{m}}(t)} .
$$

When $0<\beta_{m} \leq 1$, since $x(t)$ is strictly increasing, by Pötzsche chain rule ([3], Thm. 1.90) we obtain

$$
\begin{aligned}
\left(x^{\beta_{m}}(t)\right)^{\Delta} & =\beta_{m} \int_{0}^{1}\left[x(t)+h \mu(t) x^{\Delta}(t)\right]^{\beta_{m}-1} d h x^{\Delta}(t) \\
& =\beta_{m} \int_{0}^{1}\left[(1-h) x(t)+h x^{\sigma}(t)\right]^{\beta_{m}-1} d h x^{\Delta}(t) \\
& \geq \beta_{m}\left[x^{\sigma}(t)\right]^{\beta_{m}-1} x^{\Delta}(t) .
\end{aligned}
$$


Hence,

$$
\begin{aligned}
w_{m}^{\Delta}(t) & \leq-\rho_{m}(t) \bar{p}_{m, n-m-1}\left(t, t_{1}\right)+\rho_{m}^{\Delta}(t)\left(\frac{x^{[m]}(t)}{x^{\beta_{m}}(t)}\right)^{\sigma}-\beta_{m} \rho_{m}(t) \frac{x^{\Delta}(t)}{x^{\sigma}(t)}\left(\frac{x^{[m]}(t)}{x^{\beta_{m}}(t)}\right)^{\sigma} \\
& \leq-\rho_{m}(t) \bar{p}_{m, n-m-1}\left(t, t_{1}\right)+\rho_{m}^{\Delta}(t)\left(\frac{x^{[m]}(t)}{x^{\beta_{m}}(t)}\right)^{\sigma} .
\end{aligned}
$$

When $\beta_{m} \geq 1$, since $x(t)$ is strictly increasing, again by Pötzsche chain rule we obtain

$$
\begin{aligned}
\left(x^{\beta_{m}}(t)\right)^{\Delta} & =\beta_{m} \int_{0}^{1}\left[x(t)+h \mu(t) x^{\Delta}(t)\right]^{\beta_{m}-1} d h x^{\Delta}(t) \\
& =\beta_{m} \int_{0}^{1}\left[(1-h) x(t)+h x^{\sigma}(t)\right]^{\beta_{m}-1} d h x^{\Delta}(t) \\
& \geq \beta_{m}[x(t)]^{\beta_{m}-1} x^{\Delta}(t) .
\end{aligned}
$$

Therefore,

$$
\begin{aligned}
w_{m}^{\Delta}(t) & \leq-\rho_{m}(t) \bar{p}_{m, n-m-1}\left(t, t_{1}\right)+\rho_{m}^{\Delta}(t)\left(\frac{x^{[m]}(t)}{x^{\beta_{m}}(t)}\right)^{\sigma}-\beta_{m} \rho_{m}(t) \frac{x^{\Delta}(t)}{x(t)}\left(\frac{x^{[m]}(t)}{x^{\beta_{m}}(t)}\right)^{\sigma} \\
& \leq-\rho_{m}(t) \bar{p}_{m, n-m-1}\left(t, t_{1}\right)+\rho_{m}^{\Delta}(t)\left(\frac{x^{[m]}(t)}{x^{\beta_{m}}(t)}\right)^{\sigma} .
\end{aligned}
$$

Then, for $\beta_{m}>0$,

$$
w_{m}^{\Delta}(t) \leq-\rho_{m}(t) \bar{p}_{m, n-m-1}\left(t, t_{1}\right)+\rho_{m}^{\Delta}(t)\left(\frac{x^{[m]}(t)}{x^{\beta_{m}}(t)}\right)^{\sigma} .
$$

By using Lemma 3.2 (b) with $i=0$ and $j=0$ we see that

$$
x(t) \geq \phi_{\alpha[1, m]}^{-1}\left(x^{[m]}(t)\right) R_{m, m}\left(t, t_{1}\right)
$$

which implies

$$
\frac{x^{[m]}(t)}{x^{\beta_{m}}(t)} \leq \frac{1}{R_{m, m}^{\beta_{m}}\left(t, t_{1}\right)}
$$

Substituting (4.10) into (4.9), we get

$$
\begin{aligned}
w_{m}^{\Delta}(t) & \leq-\rho_{m}(t) \bar{p}_{m, n-m-1}\left(t, t_{1}\right)+\frac{\rho_{m}^{\Delta}(t)}{R_{m, m}^{\beta_{m}}\left(\sigma(t), t_{1}\right)} \\
& \leq-\rho_{m}(t) \bar{p}_{m, n-m-1}\left(t, t_{1}\right)+\frac{\left(\rho_{m}^{\Delta}(t)\right)_{+}}{R_{m, m}^{\beta_{m}}\left(\sigma(t), t_{1}\right)} \quad \text { for } t \in\left[t_{2}, \infty\right)_{\mathbb{T}}
\end{aligned}
$$

Integrating both sides from $t_{2}$ to $t$ we get

$$
\int_{t_{2}}^{t}\left[\rho_{m}(\tau) \bar{p}_{m, n-m-1}\left(\tau, t_{1}\right)-\frac{\left(\rho_{m}^{\Delta}(\tau)\right)_{+}}{R_{m, m}^{\beta_{m}}\left(\sigma(\tau), t_{1}\right)}\right] \Delta \tau \leq w_{m}\left(t_{2}\right)-w_{m}(t) \leq w_{m}\left(t_{2}\right),
$$

which contradicts (2.5). 
Part III: Assume that (c) holds. When $0<\beta_{m} \leq 1$, by the definition of $w_{m}(t)$, since $x(t)$ is strictly increasing, (4.7) can be written as

$$
w_{m}^{\Delta}(t) \leq-\rho_{m}(t) \bar{p}_{m, n-m-1}\left(t, t_{1}\right)+\rho_{m}^{\Delta}(t)\left(\frac{w_{m}(t)}{\rho_{m}(t)}\right)^{\sigma}-\beta_{m} \rho_{m}(t) \frac{x^{\Delta}(t)}{x^{\sigma}(t)}\left(\frac{w_{m}(t)}{\rho_{m}(t)}\right)^{\sigma} .
$$

By using Lemma $3.2(\mathrm{~b})$ with $i=0$ and $j=1$ we see that

$$
x^{[1]}(t) \geq \phi_{\alpha[2, m]}^{-1}\left(x^{[m]}(t)\right) R_{m, m-1}\left(t, t_{1}\right),
$$

which implies

$$
\begin{aligned}
\frac{x^{\Delta}(t)}{x^{\sigma}(t)} & \geq \frac{\phi_{\alpha[1, m]}^{-1}\left(x^{[m]}(t)\right)}{x^{\sigma}(t)}\left[\frac{R_{m, m-1}\left(t, t_{1}\right)}{r_{1}(t)}\right]^{1 / \alpha_{1}} \\
& \geq \frac{\phi_{\alpha[1, m]}^{-1}\left(x^{[m]}(t)\right)}{x^{\sigma}(t)}\left[\frac{R_{m, m-1}\left(t, t_{1}\right)}{r_{1}(t)}\right]^{1 / \alpha_{1}} \\
& \geq\left[\left(\frac{x^{[m]}(t)}{x^{\beta_{m}}(t)}\right)^{\sigma}\right]^{1 / \beta_{m}}\left[\frac{R_{m, m-1}\left(t, t_{1}\right)}{r_{1}(t)}\right]^{1 / \alpha_{1}} \\
& =\left[\left(\frac{w_{m}(t)}{\rho_{m}(t)}\right)^{\sigma}\right]^{1 / \beta_{m}}\left[\frac{R_{m, m-1}\left(t, t_{1}\right)}{r_{1}(t)}\right]^{1 / \alpha_{1}} .
\end{aligned}
$$

Substituting (4.13) into (4.11), we get, for $0<\beta_{m} \leq 1$,

$$
\begin{aligned}
w_{m}^{\Delta}(t) \leq & -\rho_{m}(t) \bar{p}_{m, n-m-1}\left(t, t_{1}\right)+\rho_{m}^{\Delta}(t)\left(\frac{w_{m}(t)}{\rho_{m}(t)}\right)^{\sigma} \\
& -\beta_{m} \rho_{m}(t)\left[\frac{R_{m, m-1}\left(t, t_{1}\right)}{r_{1}(t)}\right]^{1 / \alpha_{1}}\left[\left(\frac{w_{m}(t)}{\rho_{m}(t)}\right)^{\sigma}\right]^{1+1 / \beta_{m}} .
\end{aligned}
$$

When $\beta_{m} \geq 1$, by the definition of $w_{m}(t),(4.8)$ can be written as

$$
w_{m}^{\Delta}(t) \leq-\rho_{m}(t) \bar{p}_{m, n-m-1}\left(t, t_{1}\right)+\rho_{m}^{\Delta}(t)\left(\frac{w_{m}(t)}{\rho_{m}(t)}\right)^{\sigma}-\beta_{m} \rho_{m}(t) \frac{x^{\Delta}(t)}{x(t)}\left(\frac{w_{m}(t)}{\rho_{m}(t)}\right)^{\sigma} .
$$

By using Lemma 3.2 (b) with $i=0$ and $j=1$ we see that

$$
x^{[1]}(t) \geq \phi_{\alpha[2, m]}^{-1}\left(x^{[m]}(t)\right) R_{m, m-1}\left(t, t_{1}\right),
$$

which implies

$$
\begin{aligned}
\frac{x^{\Delta}(t)}{x(t)} & =\frac{x^{\Delta}(t)}{x(t)} \geq \frac{\phi_{\alpha[1, m]}^{-1}\left(x^{[m]}(t)\right)}{x(t)}\left[\frac{R_{m, m-1}\left(t, t_{1}\right)}{r_{1}(t)}\right]^{1 / \alpha_{1}} \\
& \geq \frac{\phi_{\alpha[1, m]}^{-1}\left(x^{[m]}(t)\right)}{x(t)}\left[\frac{R_{m, m-1}\left(t, t_{1}\right)}{r_{1}(t)}\right]^{1 / \alpha_{1}} \\
& =\left[\left(\frac{x^{[m]}(t)}{x^{\beta_{m}}(t)}\right)^{\sigma}\right]^{1 / \beta_{m}}\left[\frac{R_{m, m-1}\left(t, t_{1}\right)}{r_{1}(t)}\right]^{1 / \alpha_{1}} \\
& =\left[\left(\frac{w_{m}(t)}{\rho_{m}(t)}\right)^{\sigma}\right]^{1 / \beta_{m}}\left[\frac{R_{m, m-1}\left(t, t_{1}\right)}{r_{1}(t)}\right]^{1 / \alpha_{1}}
\end{aligned}
$$


Substituting (4.15) into (4.14), we get, for $\beta_{m} \geq 1$,

$$
\begin{aligned}
w_{m}^{\Delta}(t) \leq & -\rho_{m}(t) \bar{p}_{m, n-m-1}\left(t, t_{1}\right)+\rho_{m}^{\Delta}(t)\left(\frac{w_{m}(t)}{\rho_{m}(t)}\right)^{\sigma} \\
& -\beta_{m} \rho_{m}(t)\left[\frac{R_{m, m-1}\left(t, t_{1}\right)}{r_{1}(t)}\right]^{1 / \alpha_{1}}\left[\left(\frac{w_{m}(t)}{\rho_{m}(t)}\right)^{\sigma}\right]^{1+1 / \beta_{m}} .
\end{aligned}
$$

Hence, for $\beta_{m}>0$ and $t \in\left[t_{2}, \infty\right)_{\mathbb{T}}$,

$$
\begin{aligned}
w_{m}^{\Delta}(t) \leq & -\rho_{m}(t) \bar{p}_{m, n-m-1}\left(t, t_{1}\right)+\rho_{m}^{\Delta}(t)\left(\frac{w_{m}(t)}{\rho_{m}(t)}\right)^{\sigma} \\
& -\beta_{m} \rho_{m}(t)\left[\frac{R_{m, m-1}\left(t, t_{1}\right)}{r_{1}(t)}\right]^{1 / \alpha_{1}}\left[\left(\frac{w_{m}(t)}{\rho_{m}(t)}\right)^{\sigma}\right]^{1+1 / \beta_{m}} \\
\leq & -\rho_{m}(t) \bar{p}_{m, n-m-1}\left(t, t_{1}\right)+\left(\rho_{m}^{\Delta}(t)\right)+\left(\frac{w_{m}(t)}{\rho_{m}(t)}\right)^{\sigma} \\
& -\beta_{m} \rho_{m}(t)\left[\frac{R_{m, m-1}\left(t, t_{1}\right)}{r_{1}(t)}\right]^{1 / \alpha_{1}}\left[\left(\frac{w_{m}(t)}{\rho_{m}(t)}\right)^{\sigma}\right]^{1+1 / \beta_{m}} .
\end{aligned}
$$

Using Lemma 3.5 with

$$
a:=\left(\rho_{m}^{\Delta}(t)\right)_{+}, \quad b:=\beta_{m} \rho_{m}(t)\left[\frac{R_{m, m-1}\left(t, t_{1}\right)}{r_{1}(t)}\right]^{1 / \alpha_{1}}, \quad \beta:=\beta_{m} \quad \text { and } \quad u:=\left(\frac{w_{m}(t)}{\rho_{m}(t)}\right)^{\sigma}
$$

we obtain

$$
\begin{aligned}
& \left(\rho_{m}^{\Delta}(t)\right)_{+}\left(\frac{w_{m}(t)}{\rho_{m}(t)}\right)^{\sigma}-\beta_{m} \rho_{m}(t)\left[\frac{R_{m, m-1}\left(t, t_{1}\right)}{r_{1}(t)}\right]^{1 / \alpha_{1}}\left[\left(\frac{w_{m}(t)}{\rho_{m}(t)}\right)^{\sigma}\right]^{1+1 / \beta_{m}} \\
& \quad \leq\left(\frac{\beta_{m}}{\beta_{m} \rho_{m}(t)}\left[\frac{r_{1}(t)}{R_{m, m-1}\left(t, t_{1}\right)}\right]^{1 / \alpha_{1}}\right)^{\beta_{m}}\left[\frac{\left(\rho_{m}^{\Delta}(t)\right)_{+}}{1+\beta_{m}}\right]^{1+\beta_{m}} \\
& \quad=\frac{1}{\rho_{m}^{\beta_{m}}(t)}\left[\frac{\left(\rho_{m}^{\Delta}(t)\right)_{+}}{1+\beta_{m}}\right]^{1+\beta_{m}}\left[\frac{r_{1}(t)}{R_{m, m-1}\left(t, t_{1}\right)}\right]^{\beta_{m} / \alpha_{1}} .
\end{aligned}
$$

From this and from (4.17) we have

$$
w_{m}^{\Delta}(t) \leq-\rho_{m}(t) \bar{p}_{m, n-m-1}\left(t, t_{1}\right)+\frac{1}{\rho_{m}^{\beta_{m}}(t)}\left[\frac{\left(\rho_{m}^{\Delta}(t)\right)_{+}}{1+\beta_{m}}\right]^{1+\beta_{m}}\left[\frac{r_{1}(t)}{R_{m, m-1}\left(t, t_{1}\right)}\right]^{\beta_{m} / \alpha_{1}} .
$$

Integrating both sides from $t_{2}$ to $t$, we get

$$
\begin{aligned}
& \int_{t_{2}}^{t}\left[\rho_{m}(\tau) \bar{p}_{m, n-m-1}\left(\tau, t_{1}\right)\right. \\
& \left.\quad-\frac{1}{\rho_{m}^{\beta_{m}}(\tau)}\left[\frac{\left(\rho_{m}^{\Delta}(\tau)\right)_{+}}{1+\beta_{m}}\right]^{1+\beta_{m}}\left[\frac{r_{1}(\tau)}{R_{m, m-1}\left(\tau, t_{1}\right)}\right]^{\beta_{m} / \alpha_{1}}\right] \Delta \tau \leq w_{m}\left(t_{2}\right)-w_{m}(t) \leq w_{m}\left(t_{2}\right),
\end{aligned}
$$

which contradicts (2.6). 
Part IV: Assume that (d) holds. Multiplying both sides of (4.16), with $t$ replaced by $\tau$, by $H_{m}(t, \tau)$ and integrating with respect to $\tau$ from $t_{2}$ to $t \in\left[t_{2}, \infty\right)_{\mathbb{T}}$, we have

$$
\begin{aligned}
& \int_{t_{2}}^{t} \rho_{m}(\tau) \bar{p}_{m, n-m-1}\left(\tau, t_{1}\right) H_{m}(t, \tau) \Delta \tau \\
& \leq- \int_{t_{2}}^{t} H_{m}(t, \tau) w_{m}^{\Delta}(\tau) \Delta \tau \\
&+\int_{t_{2}}^{t} H_{m}(t, \tau) \rho_{m}^{\Delta}(\tau)\left(\frac{w_{m}(\tau)}{\rho_{m}(\tau)}\right)^{\sigma} \Delta \tau \\
&-\beta_{m} \int_{t_{2}}^{t} \rho_{m}(\tau) H_{m}(t, \tau)\left[\frac{R_{m, m-1}\left(\tau, t_{1}\right)}{r_{1}(\tau)}\right]^{1 / \alpha_{1}}\left[\left(\frac{w_{m}(\tau)}{\rho_{m}(\tau)}\right)^{\sigma}\right]^{1+1 / \beta_{m}} \Delta \tau .
\end{aligned}
$$

Integrating by parts and using (2.7) and (2.8), we obtain

$$
\begin{aligned}
& \int_{t_{2}}^{t} \rho_{m}(\tau) \bar{p}_{m, n-m-1}\left(\tau, t_{1}\right) H_{m}(t, \tau) \Delta \tau \\
& \leq H_{m}\left(t, t_{2}\right) w_{m}\left(t_{2}\right)+\int_{t_{2}}^{t} H_{m}^{\Delta \tau}(t, \tau) w_{m}^{\sigma}(\tau) \Delta \tau \\
&+\int_{t_{2}}^{t} H_{m}(t, \tau) \rho_{m}^{\Delta}(\tau)\left(\frac{w_{m}(\tau)}{\rho_{m}(\tau)}\right)^{\sigma} \Delta \tau \\
&-\beta_{m} \int_{t_{2}}^{t} \rho_{m}(\tau) H_{m}(t, \tau)\left[\frac{R_{m, m-1}\left(\tau, t_{1}\right)}{r_{1}(\tau)}\right]^{1 / \alpha_{1}}\left[\left(\frac{w_{m}(\tau)}{\rho_{m}(\tau)}\right)^{\sigma}\right]^{1+1 / \beta_{m}} \Delta \tau \\
& \leq H_{m}\left(t, t_{2}\right) w\left(t_{2}\right)+\int_{t_{2}}^{t}\left[\left(h_{m}(t, \tau)\right)_{-}\left(H_{m}(t, \tau)\right)^{\frac{\beta_{m}}{1+\beta_{m}}\left(\frac{w_{m}(\tau)}{\rho_{m}(\tau)}\right)^{\sigma}}\right. \\
&\left.-\beta_{m} \rho_{m}(\tau) H_{m}(t, \tau)\left[\frac{R_{m, m-1}\left(\tau, t_{1}\right)}{r_{1}(\tau)}\right]^{1 / \alpha_{1}}\left[\left(\frac{w_{m}(\tau)}{\rho_{m}(\tau)}\right)^{\sigma}\right]^{1+1 / \beta_{m}}\right] \Delta \tau .
\end{aligned}
$$

Using Lemma 3.5 with

$$
a:=\left(h_{m}(t, \tau)\right)_{-}\left(H_{m}(t, \tau)\right)^{\frac{\beta_{m}}{1+\beta_{m}}}, \quad b:=\beta_{m} \rho_{m}(\tau) H_{m}(t, \tau)\left[\frac{R_{m, m-1}\left(\tau, t_{1}\right)}{r_{1}(\tau)}\right]^{1 / \alpha_{1}},
$$

and

$$
\beta:=\beta_{m}, \quad u:=\left(\frac{w_{m}(t)}{\rho_{m}(t)}\right)^{\sigma},
$$

we get

$$
\begin{aligned}
& \left(h_{m}(t, \tau)\right)_{-}\left(H_{m}(t, \tau)\right)^{\frac{\beta_{m}}{1+\beta_{m}}}\left(\frac{w_{m}(\tau)}{\rho_{m}(\tau)}\right)^{\sigma} \\
& \quad-\beta_{m} \rho_{m}(\tau) H_{m}(t, \tau)\left[\frac{R_{m, m-1}\left(\tau, t_{1}\right)}{r_{1}(\tau)}\right]^{1 / \alpha_{1}}\left[\left(\frac{w_{m}(\tau)}{\rho_{m}(\tau)}\right)^{\sigma}\right]^{1+1 / \beta_{m}} \\
& \leq \frac{1}{\left(1+\beta_{m}\right)^{1+\beta_{m}}} \frac{\left[\left(h_{m}(t, \tau)\right)_{-}\right]^{1+\beta_{m}}}{\rho_{m}^{\beta_{m}}(\tau)}\left[\frac{r_{1}(\tau)}{R_{m, m-1}\left(\tau, t_{1}\right)}\right]^{\beta_{m} / \alpha_{1}} \\
& =\frac{1}{\rho_{m}^{\beta_{m}}(\tau)}\left[\frac{\left(h_{m}(t, \tau)\right)_{-}}{1+\beta_{m}}\right]^{1+\beta_{m}}\left[\frac{r_{1}(\tau)}{R_{m, m-1}\left(\tau, t_{1}\right)}\right]^{\beta_{m} / \alpha_{1}} .
\end{aligned}
$$


From this last inequality and from (4.18) we have

$$
\begin{aligned}
& \int_{t_{2}}^{t}\left[\rho_{m}(\tau) \bar{p}_{m, n-m-1}\left(\tau, t_{1}\right) H_{m}(t, \tau)\right. \\
& \left.\quad-\frac{1}{\rho_{m}^{\beta_{m}}(\tau)}\left[\frac{\left(h_{m}(t, \tau)\right)_{-}}{1+\beta_{m}}\right]^{1+\beta_{m}}\left[\frac{r_{1}(\tau)}{R_{m, m-1}\left(\tau, t_{1}\right)}\right]^{\beta_{m} / \alpha_{1}}\right] \Delta \tau \leq H_{m}\left(t, t_{2}\right) w_{m}\left(t_{2}\right),
\end{aligned}
$$

which implies that

$$
\begin{aligned}
& \frac{1}{H_{m}\left(t, t_{2}\right)} \int_{t_{2}}^{t}\left[\rho_{m}(\tau) \bar{p}_{m, n-m-1}\left(\tau, t_{1}\right) H_{m}(t, \tau)\right. \\
& \left.-\frac{1}{\rho_{m}^{\beta_{m}}(\tau)}\left[\frac{\left(h_{m}(t, \tau)\right)_{-}}{1+\beta_{m}}\right]^{1+\beta_{m}}\left[\frac{r_{1}(\tau)}{R_{m, m-1}\left(\tau, t_{1}\right)}\right]^{\beta_{m} / \alpha_{1}}\right] \Delta \tau \leq w_{m}\left(t_{2}\right),
\end{aligned}
$$

contradicting assumption (2.9).

Part V: Assume that (e) holds. From (4.16) we have

$$
\begin{aligned}
w_{m}^{\Delta}(t) \leq & -\rho_{m}(t) \bar{p}_{m, n-m-1}\left(t, t_{1}\right)+\rho_{m}^{\Delta}(t)\left(\frac{w_{m}(t)}{\rho_{m}(t)}\right)^{\sigma} \\
& -\beta_{m} \rho_{m}(t)\left[\frac{R_{m, m-1}\left(t, t_{1}\right)}{r_{1}(t)}\right]^{1 / \alpha_{1}}\left[\left(\frac{w_{m}(t)}{\rho_{m}(t)}\right)^{\sigma}\right]^{1+1 / \beta_{m}} \\
\leq & -\rho_{m}(t) \bar{p}_{m, n-m-1}\left(t, t_{1}\right)+\rho_{m}^{\Delta}(t)\left(\frac{w_{m}(t)}{\rho_{m}(t)}\right)^{\sigma} \\
& -\beta_{m} \rho_{m}(t)\left[\frac{R_{m, m-1}\left(t, t_{1}\right)}{r_{1}(t)}\right]^{1 / \alpha_{1}}\left[\left(\frac{w_{m}(t)}{\rho_{m}(t)}\right)^{\sigma}\right]^{1 / \beta_{m}-1}\left[\left(\frac{w_{m}(t)}{\rho_{m}(t)}\right)^{\sigma}\right]^{2} .
\end{aligned}
$$

When $0<\beta_{m} \leq 1$, in view of the definition of $w$ and (4.1), we get

$$
\left[\left(\frac{w_{m}(t)}{\rho_{m}(t)}\right)^{\sigma}\right]^{1 / \beta_{m}-1}=\left[\left(\frac{x^{[m]}(t)}{x^{\beta_{m}}(t)}\right)^{\sigma}\right]^{1 / \beta_{m}-1} \geq\left[\int_{\sigma(t)}^{\infty} \bar{p}_{m, n-m-1}\left(\tau, t_{1}\right) \Delta \tau\right]^{1 / \beta_{m}-1} .
$$

When $\beta_{m} \geq 1$, in view of the definition of $w$ and (4.2), we get

$$
\left[\left(\frac{w_{m}(t)}{\rho_{m}(t)}\right)^{\sigma}\right]^{1 / \beta_{m}-1}=\left[\left(\frac{x^{[m]}(t)}{x^{\beta_{m}}(t)}\right)^{\sigma}\right]^{1 / \beta_{m}-1} \geq\left[R_{m, m}^{\sigma}\left(t, t_{1}\right)\right]^{\beta_{m}-1} .
$$

Thus, by (4.20), (4.21), and the definition of $\delta\left(t, t_{1}\right),(4.19)$ becomes

$$
\begin{aligned}
w_{m}^{\Delta}(t) \leq & -\rho_{m}(t) \bar{p}_{m, n-m-1}\left(t, t_{1}\right)+\rho_{m}^{\Delta}(t)\left(\frac{w_{m}(t)}{\rho_{m}(t)}\right)^{\sigma} \\
& -\beta_{m} \rho_{m}(t) \delta^{\sigma}\left(t, t_{1}\right)\left[\frac{R_{m, m-1}\left(t, t_{1}\right)}{r_{1}(t)}\right]^{1 / \alpha_{1}}\left[\left(\frac{w_{m}(t)}{\rho_{m}(t)}\right)^{\sigma}\right]^{2} .
\end{aligned}
$$

Now,

$$
\begin{aligned}
& \rho_{m}^{\Delta}(t)\left(\frac{w_{m}(t)}{\rho_{m}(t)}\right)^{\sigma}-\beta_{m} \rho_{m}(t) \delta^{\sigma}\left(t, t_{1}\right)\left[\frac{R_{m, m-1}\left(t, t_{1}\right)}{r_{1}(t)}\right]^{1 / \alpha_{1}}\left[\left(\frac{w_{m}(t)}{\rho_{m}(t)}\right)^{\sigma}\right]^{2} \\
& \quad=\frac{\left(\rho_{m}^{\Delta}(t)\right)^{2}}{4 \beta_{m} \rho_{m}(t) \delta^{\sigma}\left(t, t_{1}\right)}\left[\frac{r_{1}(t)}{R_{m, m-1}\left(t, t_{1}\right)}\right]^{1 / \alpha_{1}}
\end{aligned}
$$




$$
\begin{aligned}
& -\left[\sqrt{\beta_{m} \rho_{m}(t) \delta^{\sigma}\left(t, t_{1}\right)\left[\frac{R_{m, m-1}\left(t, t_{1}\right)}{r_{1}(t)}\right]^{1 / \alpha_{1}}}\left[\left(\frac{w_{m}(t)}{\rho_{m}(t)}\right)^{\sigma}\right]\right. \\
& \left.-\frac{\rho_{m}^{\Delta}(t)}{2 \sqrt{\beta_{m} \rho_{m}(t) \delta^{\sigma}\left(t, t_{1}\right)\left[\frac{R_{m, m-1}\left(t, t_{1}\right)}{r_{1}(t)}\right]^{1 / \alpha_{1}}}}\right]^{2} \\
\leq & \frac{\left(\rho_{m}^{\Delta}(t)\right)^{2}}{4 \beta_{m} \rho_{m}(t) \delta^{\sigma}\left(t, t_{1}\right)}\left[\frac{r_{1}(t)}{R_{m, m-1}\left(t, t_{1}\right)}\right]^{1 / \alpha_{1}} .
\end{aligned}
$$

Therefore,

$$
w_{m}^{\Delta}(t) \leq-\rho_{m}(t) \bar{p}_{m, n-m-1}\left(t, t_{1}\right)+\frac{\left(\rho_{m}^{\Delta}(t)\right)^{2}}{4 \beta_{m} \rho_{m}(t) \delta^{\sigma}\left(t, t_{1}\right)}\left[\frac{r_{1}(t)}{R_{m, m-1}\left(t, t_{1}\right)}\right]^{1 / \alpha_{1}} .
$$

Integrating both sides from $t_{2}$ to $t$, we get

$$
\begin{aligned}
& \int_{t_{2}}^{t}\left[\rho_{m}(\tau) \bar{p}_{m, n-m-1}\left(\tau, t_{1}\right)\right. \\
& \left.\quad-\frac{\left(\rho_{m}^{\Delta}(\tau)\right)^{2}}{4 \beta_{m} \rho_{m}(\tau) \delta^{\sigma}\left(\tau, t_{1}\right)}\left[\frac{r_{1}(\tau)}{R_{m, m-1}\left(\tau, t_{1}\right)}\right]^{1 / \alpha_{1}}\right] \Delta \tau \leq w_{m}\left(t_{2}\right)-w_{m}(t) \leq w_{m}\left(t_{2}\right),
\end{aligned}
$$

which contradicts (2.10).

Part VI: Assume that (f) holds. Multiplying both sides of (4.22), with $t$ replaced by $\tau$, by $H_{m}(t, \tau)$ and integrating with respect to $\tau$ from $t_{2}$ to $t \in\left[t_{2}, \infty\right)_{\mathbb{T}}$, we have

$$
\begin{aligned}
& \int_{t_{2}}^{t} \rho_{m}(\tau) \bar{p}_{m, n-m-1}\left(\tau, t_{1}\right) H_{m}(t, \tau) \Delta \tau \\
& \leq-\int_{t_{2}}^{t} H_{m}(t, \tau) w_{m}^{\Delta}(\tau) \Delta \tau+\int_{t_{2}}^{t} H_{m}(t, \tau) \rho_{m}^{\Delta}(\tau)\left(\frac{w_{m}(\tau)}{\rho_{m}(\tau)}\right)^{\sigma} \Delta \tau \\
& \quad-\beta_{m} \int_{t_{2}}^{t} \rho_{m}(\tau) H_{m}(t, \tau) \delta^{\sigma}\left(\tau, t_{1}\right)\left[\frac{R_{m, m-1}\left(\tau, t_{1}\right)}{r_{1}(\tau)}\right]^{1 / \alpha_{1}}\left[\left(\frac{w_{m}(\tau)}{\rho_{m}(\tau)}\right)^{\sigma}\right]^{2} \Delta \tau
\end{aligned}
$$

Integrating by parts and using (2.7) and (2.11), we obtain

$$
\begin{aligned}
& \int_{t_{2}}^{t} \rho_{m}(\tau) \bar{p}_{m, n-m-1}\left(\tau, t_{1}\right) H_{m}(t, \tau) \Delta \tau \\
& \leq H_{m}\left(t, t_{2}\right) w_{m}\left(t_{2}\right)+\int_{t_{2}}^{t} H_{m}^{\Delta \tau}(t, \tau) w_{m}^{\sigma}(\tau) \Delta \tau+\int_{t_{2}}^{t} H_{m}(t, \tau) \rho_{m}^{\Delta}(\tau)\left(\frac{w_{m}(\tau)}{\rho_{m}(\tau)}\right)^{\sigma} \Delta \tau \\
& \quad-\beta_{m} \int_{t_{2}}^{t} \rho_{m}(\tau) H_{m}(t, \tau) \delta^{\sigma}\left(\tau, t_{1}\right)\left[\frac{R_{m, m-1}\left(\tau, t_{1}\right)}{r_{1}(\tau)}\right]^{1 / \alpha_{1}}\left[\left(\frac{w_{m}(\tau)}{\rho_{m}(\tau)}\right)^{\sigma}\right]^{2} \Delta \tau \\
& \leq H_{m}\left(t, t_{2}\right) w\left(t_{2}\right) \\
&-\int_{t_{2}}^{t}\left[\beta_{m} \rho_{m}(\tau) H_{m}(t, \tau) \delta^{\sigma}\left(\tau, t_{1}\right)\left[\frac{R_{m, m-1}\left(\tau, t_{1}\right)}{r_{1}(\tau)}\right]^{1 / \alpha_{1}}\left[\left(\frac{w_{m}(\tau)}{\rho_{m}(\tau)}\right)^{\sigma}\right]^{2}\right. \\
&\left.-\left(h_{m}(t, \tau)\right)_{-} \sqrt{H_{m}(t, \tau)}\left(\frac{w_{m}(\tau)}{\rho_{m}(\tau)}\right)^{\sigma}\right] \Delta \tau .
\end{aligned}
$$


Now,

$$
\begin{aligned}
& \beta_{m} \rho_{m}(\tau) H_{m}(t, \tau) \delta^{\sigma}\left(\tau, t_{1}\right)\left[\frac{R_{m, m-1}\left(\tau, t_{1}\right)}{r_{1}(\tau)}\right]^{1 / \alpha_{1}}\left[\left(\frac{w_{m}(\tau)}{\rho_{m}(\tau)}\right)^{\sigma}\right]^{2} \\
& -\left(h_{m}(t, \tau)\right)_{-} \sqrt{H_{m}(t, \tau)}\left(\frac{w_{m}(\tau)}{\rho_{m}(\tau)}\right)^{\sigma} \\
& =\left[\sqrt{\beta_{m} \rho_{m}(\tau) H_{m}(t, \tau) \delta^{\sigma}\left(\tau, t_{1}\right)\left[\frac{R_{m, m-1}\left(\tau, t_{1}\right)}{r_{1}(\tau)}\right]^{1 / \alpha_{1}}}\left(\frac{w_{m}(\tau)}{\rho_{m}(\tau)}\right)^{\sigma}\right. \\
& \left.-\frac{\left(h_{m}(t, \tau)\right)_{-}}{2 \sqrt{\beta_{m} \rho_{m}(\tau) \delta^{\sigma}\left(\tau, t_{1}\right)\left[\frac{R_{m, m-1}\left(\tau, t_{1}\right)}{r_{1}(\tau)}\right]^{1 / \alpha_{1}}}}\right]^{2} \\
& -\frac{\left[\left(h_{m}(t, \tau)\right)_{-}\right]^{2}}{4 \beta_{m} \rho_{m}(\tau) \delta^{\sigma}\left(\tau, t_{1}\right)}\left[\frac{r_{1}(\tau)}{R_{m, m-1}\left(\tau, t_{1}\right)}\right]^{1 / \alpha_{1}} \\
& \geq-\frac{\left[\left(h_{m}(t, \tau)\right)_{-}\right]^{2}}{4 \beta_{m} \rho_{m}(\tau) \delta^{\sigma}\left(\tau, t_{1}\right)}\left[\frac{r_{1}(\tau)}{R_{m, m-1}\left(\tau, t_{1}\right)}\right]^{1 / \alpha_{1}} \text {. }
\end{aligned}
$$

Consequently,

$$
\begin{aligned}
& \frac{1}{H_{m}\left(t, t_{2}\right)} \int_{t_{2}}^{t}\left[\rho_{m}(\tau) \bar{p}_{m, n-m-1}\left(\tau, t_{1}\right) H_{m}(t, \tau)\right. \\
& \left.-\frac{\left[\left(h_{m}(t, \tau)\right)_{-}\right]^{2}}{4 \beta_{m} \rho_{m}(\tau) \delta^{\sigma}\left(\tau, t_{1}\right)}\left[\frac{r_{1}(\tau)}{R_{m, m-1}\left(\tau, t_{1}\right)}\right]^{1 / \alpha_{1}}\right] \Delta \tau \leq w_{m}\left(t_{2}\right),
\end{aligned}
$$

which contradicts assumption (2.12).

(ii) We show that if $m=0$, then $\lim _{t \rightarrow \infty} x(t)=0$. In fact, from Lemma 3.1 we see that it is only possible when $n$ is odd. In this case,

$$
\begin{aligned}
& (-1)^{k} x^{[k]}(t)>0 \text { and } \\
& \left((-1)^{k} x^{[k]}(t)\right)^{\Delta}<0 \quad \text { for } t \in\left[t_{1}, \infty\right)_{\mathbb{T}} \text { and } k=0,1, \ldots, n-1 .
\end{aligned}
$$

Hence,

$$
\lim _{t \rightarrow \infty}(-1)^{k} x^{[k]}(t)=l_{k} \geq 0 \quad \text { for } k=0,1, \ldots, n-1 .
$$

We claim that $\lim _{t \rightarrow \infty} x(t)=l_{0}=0$. Assume that $l_{0}>0$. Then, for sufficiently large $t_{2} \in$ $\left[t_{1}, \infty\right)_{\mathbb{T}}$, we have $x\left(g_{v}(t)\right) \geq l_{0}$ for $t \geq t_{2}$. It follows that

$$
\phi_{\gamma_{v}}\left(x\left(g_{\nu}(t)\right)\right) \geq l_{0}^{\gamma_{\nu}} \geq L \quad \text { for } t \in\left[t_{2}, \infty\right)_{\mathbb{T}}
$$

where $L:=\min _{v=0}^{N}\left\{l_{0}^{\gamma_{v}}\right\}>0$. Then from (1.1) we obtain

$$
-\left(x^{[n-1]}(t)\right)^{\Delta} \geq L \sum_{\nu=0}^{N} p_{\nu}(t)=L \hat{p}_{0}(t) .
$$


Integrating this from $t$ to $v \in[t, \infty)_{\mathbb{T}}$, we get

$$
-x^{[n-1]}(v)+x^{[n-1]}(t) \geq L \int_{t}^{v} \hat{p}_{0}(\tau) \Delta \tau
$$

and by $(4.23)$ we see that $x^{[n-1]}(v)>0$. Hence, by taking limits as $v \rightarrow \infty$ we have

$$
x^{[n-1]}(t) \geq L \int_{t}^{\infty} \hat{p}_{0}(\tau) \Delta \tau
$$

If $\int_{t}^{\infty} \hat{p}_{0}(\tau) \Delta \tau=\infty$, then we have reached a contradiction. Otherwise,

$$
\left(x^{[n-2]}(t)\right)^{\Delta} \geq L^{1 / \alpha_{n-1}}\left[\frac{1}{r_{n-1}(t)} \int_{t}^{\infty} \hat{p}_{0}(\tau) \Delta \tau\right]^{1 / \alpha_{n-1}}=L^{1 / \alpha_{n-1}} \hat{p}_{1}(t) .
$$

Integrating this from $t$ to $v \in[t, \infty)_{\mathbb{T}}$ and letting $v \rightarrow \infty$, by (4.23) we get

$$
-x^{[n-2]}(t) \geq L^{1 / \alpha_{n-1}} \int_{t}^{\infty} \hat{p}_{1}(\tau) \Delta \tau .
$$

If $\int_{t}^{\infty} \hat{p}_{1}(\tau) \Delta \tau=\infty$, then we have reached a contradiction. Otherwise,

$$
-\left(x^{[n-3]}(t)\right)^{\Delta} \geq L^{1 / \alpha[n-2, n-1]}\left[\frac{1}{r_{n-2}(t)} \int_{t}^{\infty} \hat{p}_{1}(\tau) \Delta \tau\right]^{1 / \alpha_{n-2}}=L^{1 / \alpha[n-2, n-1]} \hat{p}_{2}(t) .
$$

Continuing this process, we get

$$
-x^{[1]}(t) \geq L^{1 / \alpha[2, n-1]} \int_{t}^{\infty} \hat{p}_{n-2}(\tau) \Delta \tau
$$

If $\int_{t}^{\infty} \hat{p}_{n-2}(\tau) \Delta \tau=\infty$, then we have reached a contradiction. Otherwise,

$$
-x^{\Delta}(t) \geq L^{1 / \alpha[1, n-1]}\left[\frac{1}{r_{1}(t)} \int_{t}^{\infty} \hat{p}_{n-2}(\tau) \Delta \tau\right]^{1 / \alpha_{1}}=L^{1 / \alpha} \hat{p}_{n-1}(t)
$$

Again, integrating from $t_{2}$ to $t \in\left[t_{2}, \infty\right)_{\mathbb{T}}$, we get

$$
-x(t)+x\left(t_{2}\right) \geq L^{1 / \alpha} \int_{t_{2}}^{t} \hat{p}_{n-1}(\tau) \Delta \tau
$$

If $\int_{t}^{\infty} \hat{p}_{n-1}(\tau) \Delta \tau=\infty$, then we have $\lim _{t \rightarrow \infty} x(t)=-\infty$, which contradicts the assumption that $x(t)>0$ eventually. This shows that if $m=0$, then $\lim _{t \rightarrow \infty} x(t)=0$. This completes the proof.

Competing interests

The author declares that he has no competing interests.

This work was supported by Research Deanship of Hail University under grant No. 0150287. 


\section{References}

1. Chen, DX, Qu, PX: Oscillation of even order advanced type dynamic equations with mixed nonlinearities on time scales. J. Appl. Math. Comput. 44(1-2), 357-377 (2014)

2. Zhang, SY, Wang, QR, Kong, Q: Asymptotics and oscillation of nth-order nonlinear dynamic equations on time scales. Appl. Math. Comput. 275, 324-334 (2016)

3. Bohner, M, Peterson, A: Dynamic Equations on Time Scales: An Introduction with Applications. Birkhäuser, Boston (2001)

4. Bohner, M, Peterson, A (eds.): Advances in Dynamic Equations on Time Scales. Birkhäuser, Boston (2003)

5. Grace, SR, Agarwal, R, Zafer, A: Oscillation of higher order nonlinear dynamic equations on time scales. Adv. Differ Equ. 2012, 67 (2012)

6. Wu, X, Sun, T, Xi, H, Chen, C: Kamenev-type oscillation criteria for higher-order nonlinear dynamic equations on time scales. Adv. Differ. Equ. 2013, 248 (2013)

7. Sun, T, Yu, W, He, Q: New oscillation criteria for higher order delay dynamic equations on time scales. Adv. Differ. Equ. $2014,328(2014)$

8. Sun, $T, H e, O, X i, H, Y u, W$ : Oscillation for higher order dynamic equations on time scales. Abstr. Appl. Anal. 2013, Article ID 268721 (2013)

9. Hassan, TS, Kong, Q: Asymptotic and oscillatory behavior of $n$ th-order half-linear dynamic equations. Differ. Equ. Appl. 6(4), 527-549 (2014)

10. Grace, SR, Hassan, TS: Oscillation criteria for higher order nonlinear dynamic equations. Math. Nachr. 287(14-15), 1659-1673 (2014)

11. Hassan, TS: Oscillation criteria for higher order quasilinear dynamic equations with Laplacians and a deviating argument. J. Egypt. Math. Soc. Available online 25 November 2016

12. Huang, $\mathrm{XY}$ : Oscillatory behavior of $\mathrm{N}$-th-order neutral dynamic equations with mixed nonlinearities on time scales. Electron. J. Differ. Equ. 2016, 16 (2016)

13. Erbe, $L$, Mert, R, Peterson, A, Zafer, A: Oscillation of even order nonlinear delay dynamic equations on time scales. Czechoslov. Math. J. 63(138)(1), 265-279 (2013)

14. Mert, R: Oscillation of higher-order neutral dynamic equations on time scales. Adv. Differ. Equ. 2012, 68 (2012)

15. Sun, $T, Y u, W, X i, H$ : Oscillatory behavior and comparison for higher order nonlinear dynamic equations on time scales. J. Appl. Math. Inform. 30(1-2), 289-304 (2012)

16. Karpuz, B: Unbounded oscillation of higher-order nonlinear delay dynamic equations of neutral type with oscillating coefficients. Electron. J. Qual. Theory Differ. Equ. 2009, 34 (2009)

17. Erbe, L, Karpuz, B, Peterson, A: Kamenev-type oscillation criteria for higher-order neutral delay dynamic equations. Int. J. Difference Equ. 6(1), 1-16 (2011)

18. Sun, Y, Hassan, TS: Comparison criteria for odd order forced nonlinear functional neutral dynamic equations. Appl. Math. Comput. 251, 387-395 (2015)

19. Erbe, L, Jia, B, Peterson, A: Oscillation of $n$th order superlinear dynamic equations on time scales. Rocky Mt. J. Math. 41(2), 471-491 (2011)

20. O'Regan, D, Hassan, TS: Oscillation criteria for solutions to nonlinear dynamic equations of higher order. Hacet. J. Math. Stat. 45(2), 417-427 (2016)

21. Tunç, E: Oscillation results for even order functional dynamic equations on time scales. Electron. J. Qual. Theory Differ. Equ. 2014, 27 (2014)

22. Zhang, C, Agarwal, RP, Li, T: Oscillation and asymptotic behavior of higher-order delay differential equations with p-Laplacian like operators. J. Math. Anal. Appl. 409(2), 1093-1106 (2014)

23. Džurina, J, Baculíková, B: Oscillation and asymptotic behavior of higher-order nonlinear differential equations. Int. J. Math. Math. Sci. 2012, Article ID 951898 (2012)

24. Zhang, C, Li, T, Agarwal, RP, Bohner, M: Oscillation results for fourth-order nonlinear dynamic equations. Appl. Math Lett. 25(12), 2058-2065 (2012)

25. Agarwal, RP, Bohner, M, Li, T, Zhang, C: A Philos-type theorem for third-order nonlinear retarded dynamic equations Appl. Math. Comput. 249, 527-531 (2014)

26. Hassan, TS: Comparison criterion for even order forced nonlinear functional dynamic equations. Commun. Appl. Anal. 18, 109-122 (2014)

27. Agarwal, RP, Grace, SR, Hassan, TS: Oscillation criteria for higher order nonlinear functional dynamic equations with mixed nonlinearities. Commun. Appl. Anal. 19, 369-402 (2015)

28. Hassan, TS, Grace, SR: Comparison criteria for nonlinear functional dynamic equations of higher order. Discrete Dyn. Nat. Soc. 2016, Article ID 6847956 (2016)

29. Saker, SH: Oscillation criteria of second-order half-linear dynamic equations on time scales. J. Comput. Appl. Math. $177,375-387$ (2005)

30. Beckenbach, EF, Bellman, R: Inequalities. Springer, Berlin (1961)

31. Sun, YG, Wong, JS: Oscillation criteria for second order forced ordinary differential equations with mixed nonlinearities. J. Math. Anal. Appl. 334, 549-560 (2007)

32. Hassan, TS, Kong, Q: Interval criteria for forced oscillation of differential equations with $p$-Laplacian, damping, and mixed nonlinearities. Dyn. Syst. Appl. 20, 279-294 (2011)

33. Hassan, TS, Erbe, L, Peterson, A: Forced oscillation of second order functional differential equations with mixed nonlinearities. Acta Math. Sci. Ser. B 31, 613-626 (2011)

34. Ozbekler, A, Zafer, A: Oscillation of solutions of second order mixed nonlinear differential equations under impulsive perturbations. Comput. Math. Appl. 61, 933-940 (2011)

35. Zhang, S, Wang, Q: Oscillation of second-order nonlinear neutral dynamic equations on time scales. Appl. Math. Comput. 216(10), 2837-2848 (2010) 